\title{
Robust and Efficient Field Features Detection for Localization
}

\author{
D. Herrero-Pérez and H. Martínez-Barberá \\ Dept. Information and Communication Engineering, \\ University of Murcia, 30100 Murcia, Spain \\ dherrero@dif.um.es, humberto@um.es
}

\begin{abstract}
In some Robocup leagues, specially in the four-legged league, robots make use of coloured landmarks for localisation. Because these landmarks have no correlation with real soccer, it seems a natural approach to remove them. But for this to be a reality, there are some difficulties that need to be solved, mainly an efficient and robust field features detection and an efficient localisation technique to manage such type of information. In this paper we deal with an approach for field features detection based on finding intersections between field lines which runs at frame rate in the AIBO robots. We also present some experimental results of the vision system and a comparison of the traditional coloured landmark localisation and the field features only localisation, both using a fuzzy-Markov localisation technique.
\end{abstract}

Keywords: Autonomous robots, fuzzy logic, image processing, localisation, state estimation.

\section{Introduction}

The Sony Four-Legged Robot (SFLR) League is one of the official leagues in Robocup, in which a standardised robot platform is used, the Sony AIBO. The main exteroceptive sensor is a camera, which can detect objects on the field. Objects are colour coded: there are four uniquely coloured landmarks, two goal nets of different colour, the ball is orange, and the robots wear coloured uniforms. However, in a real soccer field there are not characteristic coloured cues. The rules of RoboCup are gradually changed year after year in order to push progress towards the final goal. Removal of the artificial coloured beacons will be the next step in this direction.

Moreover, coloured landmarks and nets are not frequently perceived in game's conditions, because robots are constantly looking to field to find the ball. Therefore, natural landmarks over the field are constantly perceived, that is field lines, which can be used to update robot localisation more frequently. For these field lines to be successfully used, there are two problems that must be addressed: robust field features detection in real time, and robust localisation able to manage such information. 
Preliminary work has been done by some teams in this league to allow the robot to self-localise without using the artificial beacons. For instance, the German Team and other teams use a sub-sampling technique to detect pixels that belong to the field lines [6], distinguishing between field lines along the field and field lines across the field to improve the goalkeepers localisation. These pixels are used in a Monte-Carlo localisation (MCL) schema [5]. Some teams use a kalman filter as their localisation approach, although it can not handle more than one position hypothesis. In order to overcome this problem, rUNSWift uses a Multiple Hypothesis Tracking approach called the multi-hypothesis localisation (MHL) [4. In our case, we are using a fuzzy-Markov self-localisation technique, in which the robot location is modelled as a belief distribution on a $2 \frac{1}{2} \mathrm{D}$ possibility grid [1]. This formalism allows us to represent and track multiple possible positions where the robot might be. Moreover, it only requires an approximate model of the sensor system and a qualitative estimate of the robot's displacement.

In this paper we propose an alternative solution to using field line detection for the localisation process, which is based on field line intersections detection. The proposed method is described in section 2. These detected features can be introduced in any localisation filter, as those mentioned above. Section 3 describes briefly how we introduce these perceptions in a fuzzy-Markov localisation filter. Section 4 presents some experimental results of the proposed method by way of the evaluation of the localisation accuracy. Finally, conclusions are presented.

\section{Perception}

The AIBO robots use a CCD camera as the main exteroceptive sensor. The perception process is in charge of extracting convenient features of the environment from the images provided by the camera. As the robot will localise relying on the extracted features, both the amount of features detected and their quality will clearly affect the process. Because of the league rules, all the processing must be done on board and for practical reasons it has to be performed in real time, which prevents us from using time consuming algorithms.

A typical approach for detecting straight lines in digital images is the Hough Transform and its numerous variants. The various variants have been developed to try to overcome the major drawbacks of the standard method, namely, its high time complexity and large memory requirements. Instead of using the field lines as references for the self-localisation, we use the corners produced by the intersection of the field lines (which are white). The two main reasons for using corners is that they can be labelled (depending on the type of intersection) and they can be tracked more appropriately given the small field of view of the camera.

\subsection{Vision System Description}

The vision system flowchart that we use on the AIBOs is depicted in Fig. 1. The source is the YUV images from the camera and the result is a set of features 


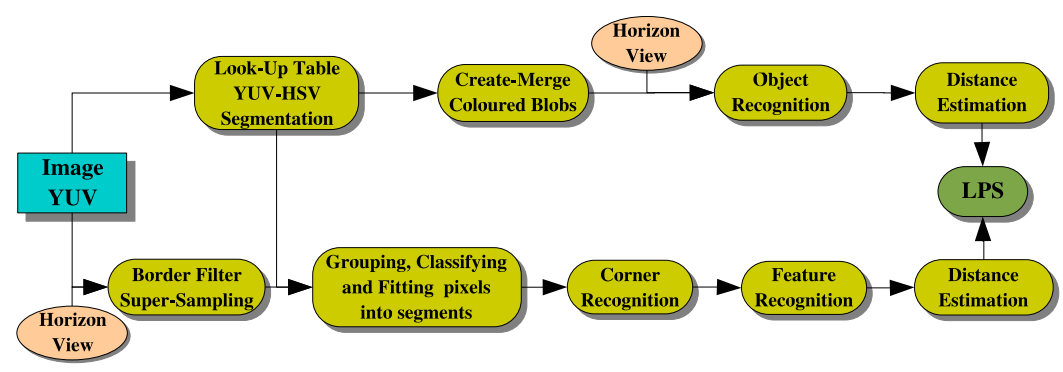

Fig. 1. Vision system flowchart

of the environment in robot-centric coordinates that is called Local Perceptual Space (LPS). There are two different paths: the detection of colour-coded object (ball, nets, robots, and landmarks) and the detection of field features (field line intersections). We will describe the second one.

The first step after taking a camera image is computing the horizon line, based on the pose of the robot and its head. The idea is looking for field lines only on the horizontal plane, thus saving computing time and avoiding false positives.

The second step is applying a border extraction filter to the brightness channel of the YUV image, in particular a Sobel filter is used (Figs. 2]b and 3b). By sub-sampling the border-filtered image from the bottom up to the horizon line (Figs. 2a and 3a), transitions of the form non-border (black) $\rightarrow$ border (white) $\rightarrow$ non-border (black) are stored and considered for further processing.

The third step, which is shared with the coloured objects recognition path, is converting the YUV image to the HSV colour space (Figs. 20 and 3r). As this is a time consuming process, we perform the conversion using a look-up table with pre-computed values.

The fourth step is filtering out non-field line transitions from the candidate list. These candidate transitions are checked with the HSV colour segmented image to detect which ones have been produced by the field lines, in particular we consider the following transition types:

- Carpet-to-line. Transition from carpet (green) pixels to line (white) pixels.

- Line-to-carpet. Transition from line (white) pixels to carpet (green) pixels.

- Carpet-to-net. Transition from carpet (green) pixels to net pixels (cyan or yellow).

These labelled transitions are grouped together into sets of transitions that belong to the same straight segment. These segments are obtained using the Recursive Iterative End Point Fit Algorithm (RIEPFA) 2]. RIEPFA groups a set of points into segments by evaluating the distance of the points to candidate end segment points, which are recursively divided into smaller segments until the fit criteria is hold (point to segment distance). Segments are further evaluated to meet a minimum number of points and maximum point-to-point distance 


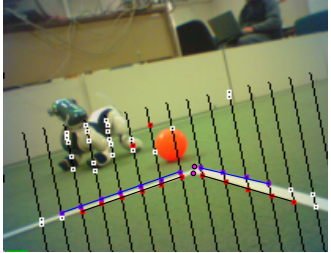

(a)

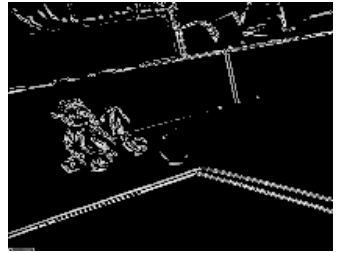

(b)

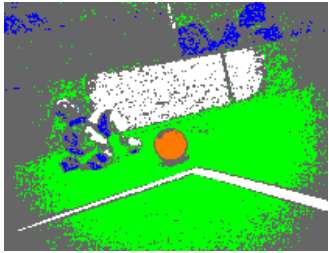

(c)

Fig. 2. Image from the goalkeeper position (a) Sub-sampling and intersections. (b) Sobel filter of the YUV brightness channel. (c) Colour segmentation of the HSV image.

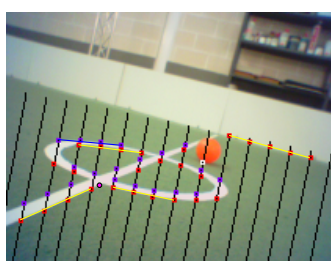

(a)

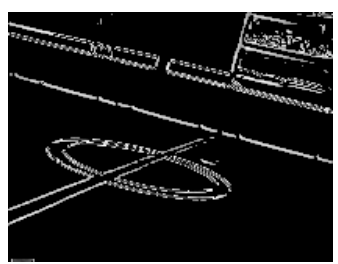

(b)

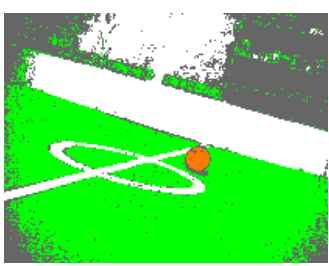

(c)

Fig. 3. Image from the defender position (a) Sub-sampling and intersections. (b) Sobel filter of the YUV brightness channel. (c) Colour segmentation of the HSV image.

criteria. The segments that are not rejected by the previous criteria are then labelled according to the transition pixels that originated the segment.

The fifth step is finding intersections between the labelled segments to produce field lines corners (Figs. 2a and 3 a). The intersections are evaluated based on the segments labelling, segments orientation, and end points proximity. Valid intersections are then labelled as following:

- Closed-intersection. Intersection between carpet-to-line or line-to-carpet segments, with an angle less than 180 degrees.

- Open-intersection. Intersection between carpet-to-line or line-to-carpet segments, with an angle greater than 180 degrees.

- Net-intersection. Intersection between a carpet-to-line or line-to-carpet segment and a carpet-to-net segment. The angle is not taken into account.

The sixth step is grouping segment intersections into labelled field features, rejecting those that do not lie into any of the following categories:

- Type C. An open-intersection nearby of a closed-intersection. This feature can be found in the corners of the goal keeper area.

- Type T-field. Two closed-intersections. This feature can be found in the intersection of the field lines perimeter and any other type of line.

- Type T-net. A closed-intersection nearby of a net-intersection. This feature can be found in the intersection between the goal lines and the corresponding net. 
The seventh step is the last one in the pipeline. It consists on computing the distance and orientation from the robot to each detected field feature. The pose of the camera is computed using the joint angles of the legs and the head. Then the pixel from the image that represents a field feature is projected onto the field, subject to the constraint that the feature is on the horizontal ground plane. Then the projection point is used to compute the corresponding distance and orientation.

\subsection{Vision System Analysis}

In this section we will discuss two important aspects of the field features detection procedure presented above: robustness and performance. Because the detected features will be used for locating the robot on the field, one key point is false positives. Reducing the possibility of false positives reduces the possibility of wrong position estimation. The goal of the presented detection procedure is the identification of field lines intersection, and the underlying idea is the filtering of candidates that might be false positives.

Several works detects transitions in segmented images [5] or extracts corners from grey-scale images [3]. But these approaches can not distinguish pixels belonging to the lines or to white robots. Although, these techniques can not distinguish pixels belonging to straight-lines or curve-lines. Furthermore, in the case the carpet perimeter is placed over a plain floor or there is a white hurdle, these pixels are labelled as lines. Thus, typical false positive cases are produced by the white robots, non-straight lines and non-normalised transition in the field perimeter.

All these cases can produce false positives what are very difficult to avoid without evaluate if the pixel belong to a straight line or not. Other technique to get straight line segments is the Hough Transform, but it has as main disadvantage the high computational cost.

\section{Localisation}

Once we are able to obtain a series of percept, be them coloured landmarks, nets or field features, we need a way to combine such information in order to estimate the robots pose on the field: the localisation filter. Although other techniques might be used, without loss of generality we describe how we model the uncertainty associated to the perceived field features using a fuzzy-Markov technique [1]. See [3] for more details.

\section{Experimental Results}

In order to validate the perception process described in the paper, two localisation experiments have been performed. In both cases the robot has been placed in the goal area, facing more or less to the opposite net. This is the typical goalkeeper position. For a goalkeeper localisation is critical because many behaviour 
depend on the absolute position. If localisation fails, the robot would start wandering around, possibly leaving the net clear to the other team. In both cases, the localisation process is initialised with a belief distributed along the whole field, that is it does not know its own location. Then the robot starts scanning its surroundings by moving its head from left to right. As soon as a feature is perceived, it is incorporated into the localisation process. The first experiment corresponds to the standard RoboCup scenario, with coloured landmarks and nets as the only perceptual source for localisation. The second experiment corresponds to a possible future RoboCup scenario, in which coloured landmarks have been completely removed, being the perceptual sources the nets and the field lines.

In the experiments we compare the estimated position with the real position. In order to measure the real robot position (actually tracking many robots and the ball) we use an external vision system. This is composed of an overhead camera with a wide angle lens mounted on a aluminium structure at 2.5 metres over the floor. The robot wears a coloured mark which allows computing both the position and orientation of the robot. Because of the high distortion of the lens, the accuracy of the position is of \pm 2 centimetres and \pm 5 degrees.

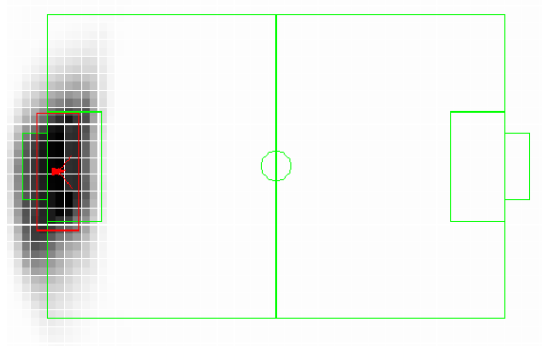

(a)

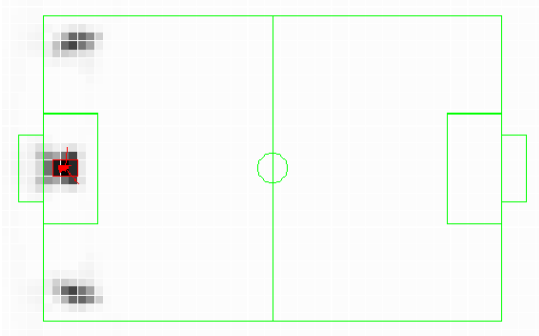

(b)

Fig. 4. Resulting beliefs (a) Coloured landmarks and nets (b) Field lines and nets

The experiments start with belief distributed along the whole field and the robots are left for some seconds scanning the surroundings. The resulting beliefs of both experiments are shown in Fig. 4. The red box corresponds to the estimate of the uncertainty of the robot's position (obtained by finding the bounding box of the highest possibility area) and the red arrow corresponds to the estimate of the robot's position (obtained by defuzzification of the fuzzy belief with the centre of gravity). The comparison of the belief distributions shows that the uncertainty obtained in the standard RoboCup experiment (Fig. 4a) is larger than the one obtained with field lines (Fig 4 $\mathrm{b}$ ). This is because the coloured landmarks are further from the robot than the goal area lines are, thus having a larger uncertainty in the distance. Moreover, this uncertainty in the distance also depends on the colour calibration (the more pixels are segmented the closer the landmark will be perceived), while the field features are less sensible to this effect. 


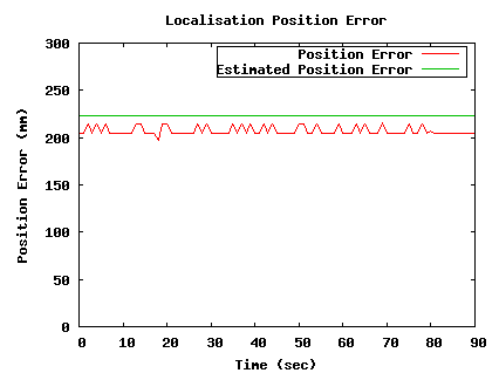

(a)

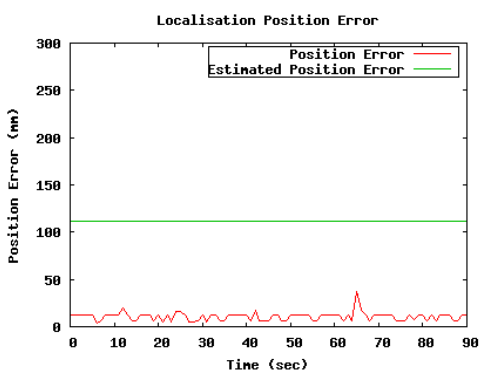

(b)

Fig. 5. Position error (a) using coloured landmarks and nets and (d) using field lines and nets

The reduction of the uncertainty conditions very much the accuracy of the localisation process, that is, less uncertainty usually leads to better accuracy. Fig. 5 shows the absolute position error over time, both for the coloured landmarks and field lines experiments. The absolute error is computed taking the difference between the estimated position (on-board) and the measured position (overhead camera). The estimated absolute error is computed from the uncertainty of the belief distribution and the estimated position (on-board). Because the belief distribution is a grid, the minimum estimated absolute error corresponds to the tessellation size, which corresponds to $100 \mathrm{~mm}$ in these experiments.

\section{Conclusions}

This paper presents a novel technique for recognising field features for localisation in the RoboCup Four-Legged League (FLL), although the results can be used in other leagues and scenarios. There is a push towards the removal of coloured landmarks in RoboCup leagues to make the fields more soccer-like. The typical approach in this context is the recognition of field lines. The described technique is based on the recognition of field line intersections, keeping into main the constraints of the FLL: on-board processing and the AIBO's camera. The computational burden of the process is low, and it can be set to run at frame rate on the AIBOs. In addition, most of the efforts of the technique are in the direction of avoiding false positives, which can lead to wrong localisation.

In order to validate the usability of the proposed vision process, the detected features are incorporated in a localisation filter. Without loss of generality, we use a fuzzy-markov grid in which we have modelled the perception and its associated uncertainty of different types of field line intersections. This localisation filter provides an effective solution to the problem of localisation of a legged robot in the RoboCup domain [1].

We have presented a series of experiments to show the performance of the localisation process in the standard RoboCup field (with uniquely coloured landmarks and nets) and the more soccer-like scenario (with field lines and uniquely 
coloured nets). We have compared the estimated robot position with the real one in both scenarios and have shown the feasibility of the removal of the landmarks. Moreover, in certain circumstances the field lines localisation can be more accurate than using landmarks (at least from the goalkeeper's position).

The standard RoboCup scenario provides unique coloured landmarks (that is, there is no ambiguity in the perception process), while the field lines scenario needs unique nets to cope with the natural symmetry of the field. Currently there are no plans to move from unique nets to equally coloured-nets (at least in the FLL), and thus this is not a drawback of the proposed method. Once the use of field lines and unique nets localisation is common in the leagues, there will be necessary any other means to break with the ambiguity, which are left as future work.

\section{Acknowledgements}

This work has been supported by CICYT project DPI2004-07993-C03-02.

\section{References}

1. Buschka, P., Saffiotti, A., Wasik, Z.: Fuzzy landmark-based localization for a legged robot. In: Intelligent Robots and Systems (IROS), Takamatsu, Japan, pp. 1205$1210(2000)$

2. Duda, R., Hart, R.y.: Classification and scene analysis. John Wiley and Sons, Chichester (1973)

3. Herrero-Pérez, D., Martínez-Barberá, H., Saffiotti, A.: Fuzzy self-localization using natural features in the four-legged league. In: RoboCup 2003, pp. 110-121 (2004)

4. Jensfelt, P., Kristensen, S.: Active global localization for a mobile robot using multiplehypothesis tracking. IEEE Transactions on Robotics and Automation 17(5), 748-760 (2001)

5. Röfer, T., Jüngel, M.: Fast and robust edge-based localization in the sony fourlegged robot league. In: RoboCup 2003, Padova, Italy (2004)

6. Röfer, T., Laue, T., Thomas, D.: Particle-filter-based self-localization using landmarks and directed lines. In: Bredenfeld, A., Jacoff, A., Noda, I., Takahashi, Y. (eds.) RoboCup 2005. LNCS (LNAI), vol. 4020, Springer, Heidelberg (2006) 